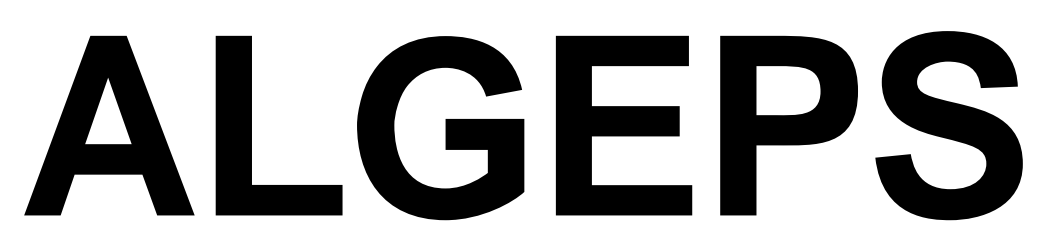

REVISTA DE GEOLOGIA, SĖRIE B no 577 - Gener del 2012

ISSN $1132-7014$

D.L.B. 28.178 - 92

12 pàgines

RECORRIDO DESDE VALMADRID A LA PUEBLA DE ALBORTÓN, A FUENDETODOS Y A AZUARA, A TRAVÉS DEL PATRIMONIO GEOLÓGICO Y MINERO DE LAS COMARCA DEL CAMPO DE BELCHITE

Josep M. Mata-Perelló

Aquest recorregut va ésser experimentat amb docents el dia 1 DE MAIG DEL 2010 


\title{
RECORRIDO DESDE VALMADRID A LA PUEBLA DE ALBORTÓN, A FUENDETODOS Y A AZUARA, A TRAVÉS DEL PATRIMONIO GEOLÓGICO Y MINERO DE LAS COMARCA DEL CAMPO DE BELCHITE
}

\author{
Por Josep M. Mata-Perelló
}

\section{ADVERTENCIAS PREVIAS}

Como en otros recorridos de RECONOCIMIENTO GEOLÓGICO (o de RECONOCIMIENTO GEOLÓGICO Y MINERO), el recorrido se compondrá de diversas PARADAS. En este caso serán seis.

Por otra parte, habrá que tener en cuenta, en todo momento, especialmente antes de empezar los recorridos de los diferentes tramos, el estado de los caminos y carreteras, por donde transitará el recorrido. Al respecto, cabe decir que prácticamente todos estos tramos se halla en buenas condiciones.

Finalmente, como ya hacemos en otros recorridos similares, queremos decir que hace falta tener un cuidado muy especial en el respeto a la naturaleza, a lo largo de todo el recorrido del itinerario, y también fuera de él.

\section{BREVE INTRODUCCIÓN GEOLÓGICA}

El recorrido de este itinerario, se desarrollará exclusivamente por una de las tres unidades geológicas que constituyen el suelo y el subsuelo de Aragón. Concretamente por la Depresión Geológica del Ebro (en donde se iniciarla y finalizara el recorrido del itinerario).

Así, a lo largo de todo de todo el recorrido del itinerario, se irán encontrando afloramientos cenozoicos, en su mayoría de carácter arcilloso y calcolutítico, pertenecientes al Mioceno. De entre estos materiales, cabe destacar los afloramientos carbonatados y los yesosos, explotados en diversos lugares del recorrido.

Asimismo, a menudo, encontraremos afloramientos de los materiales detríticos cuaternarios, por encima de los anteriores

\section{BREVE INTRODUCCIÓN GEOGRÁFICA}

El recorrido del presente itinerario se efectuará exclusivamente por una comarca aragonesa, la del Campo de Belchite. Así, se iniciará en la población de Valmadrid, para terminar en las cercanías de Azuara. 
En este recorrido, se circulará por los términos municipales de: Valmadrid, la Puebla de Albortón, Fuendetodos y Azuara, todos ellos de la comarca del Campo de Belchite.

\section{OBJETIVOS GENERALES DE ESTE ITINERARIO}

En este itinerario, los objetivos generales que se han de conseguir, se pueden concretar en los siguientes aspectos:

1.- Estudio y reconocimiento de los materiales cenozoicos (en buena parte del Mioceno) situados en la Depresión Geológica del Ebro, que iremos encontrando a lo largo del recorrido del itinerario, entre las inmediaciones de Valmadrid y las de la población de Azuara.

2.- Visión de algunas de las antiguas explotaciones encontradas a lo largo del recorrido del itinerario. En concreto de;

2A) de diversas explotaciones de yeso, situadas en los municipios de Valmadrid y de la Puebla de Albortón.

2B) de diversas explotaciones de calizas: las explotaciones de la Puebla de Albortón y las de Azuara, activas en estos momentos.

3.- Observación del impacto producido por las actividades mineras. I si se da el caso, de las restauraciones realizadas para paliar este impacto.

4.- Visión de los diferentes lugares directamente relacionados con el Patrimonio Geológico y Minero que iremos encontrando a lo largo del recorrido del presente itinerario

\section{ANTECEDENTES BIBLIOGRÁFICOS}

En relación con este itinerario, no conocemos ningún antecedente, relativo a otro itinerario que discurra por este lugar. En este sentido, este itinerario constituye un antecedente, si no estamos equivocados.

Por otra parte, haremos mención de algunos trabajos, de carácter geológico generalista, que corresponden a los trabajos del IGME (1972, 1974 y 1975), relativos al Mapa Geológico de España (a Escala 1.200.000), al Mapa Metalogenético de España y al Mapa de Rocas Industriales de España

Con respecto a las mineralizaciones que iremos encontrando, mencionaremos los trabajos de: CALVO (2001), CALVO et altri (1988); MAESTRE (1845); así como nuestros trabajos: MATA-PERELLÓ (1987 y 1998). 
También mencionaremos el trabajo de PRAMES (2005) dedicado a la comarca del Campo de Belchite. Así como el del GOBIERNO DE ARAGÓN (2001), dedicado a los Puntos de Interés Geológico de Aragón.

Finalmente, diremos que todos estos trabajos (así como otros que ahora no hemos aludido), figurarán mencionados, por orden alfabético, en el apartado dedicado a las REFERENCIAS BIBLIOGRÁFICAS.

\section{RECORRIDO DEL ITINERARIO}

Este recorrido se iniciará en las inmediaciones del pueblo de Valmadrid. En torno a ese pueblo se hará una parada. Luego, el recorrido se dirigirá hacia la población de la Puebla de Albortón, en donde se realizaran dos nuevas paradas.

Tras ello, el recorrido se dirigirá hacía Fuendetodos, realizándose una nueva parada en la vieja estación de Azuara. Tras ello, en Fuendetodos se realizará una nueva parada.

Tras ello, el recorrido se dirigirá hacía Azuara, realizándose una nueva parada antes de llegar al pueblo, la última del itinerario.

\section{DESCRIPCIÓN DEL ITINERARIO}

Como de costumbre, haremos una serio de PARADAS (o ESTACIONES), en donde se realizaran diversas explicaciones en torno a las características del lugar en donde se halla la PARADA. Por otra parte, en ellas haremos mención del término municipal dónde se encuentran, así como del número del "Mapa Topográfico Nacional (a escala 1:50.000, que indicaremos entre paréntesis. Así, ahora (en este recorrido) utilizaremos solamente cuatro hojas: concretamente la 411 (o de Longares), $\mathbf{4 1 2}$ (o de Pina de Ebro), 439 (o de Azuara) y la $\mathbf{4 4 0}$ (o de Belchite).

Así, la relación ordenada de las paradas que constituyen el recorrido de este itinerario, es la siguiente:

PARADA 1. HORNO DE YESO, (término municipal del Valmadrid, comarca del Campo de Belchite). (Hoja 411).

El recorrido del presente itinerario lo iniciaremos en la localidad de Valmadrid, la más septentrional de la comarca del Campo de Belchite. Concretamente, lo iniciaremos a la entrada de la población, viniendo desde la Torrecilla de Valmadrid y de Zaragoza.

Este lugar se halla en plena Depresión Geológica del Ebro. Así, los materiales que afloran por doquier pertenecen al cenozoico y en concreto al Mioceno. Entre estos 
materiales se observan niveles de yesos, de arcillas, calcolutitas, areniscas y de niveles carbonatados. Por encima de estos materiales, muy a menudo hay afloramientos de materiales detríticos cuaternarios.

En el lugar de la parada se hacen ostensibles unos afloramientos de niveles yesosos miocénicos. Y precisamente, sobre estos materiales ha habido una pequeña explotación de yeso, construyéndose un horno de yeso, sobre estos mismos materiales. Este horno forma parte del patrimonio minero de esta comarca.

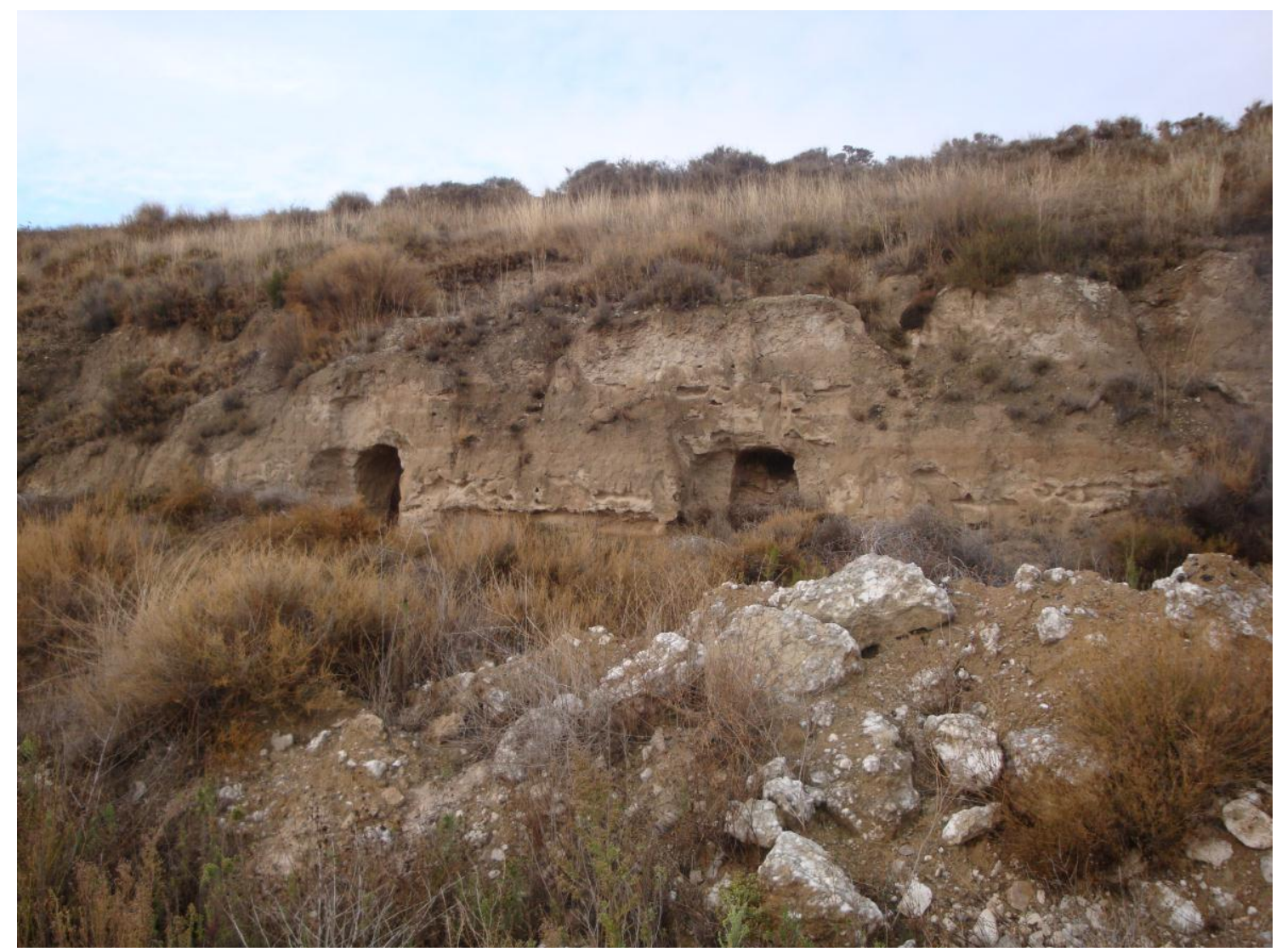

EL HORNO Y EL YESO

PARADA 2. HORNOS DE YESO DE LA PUEBLA DE ALBORTÓN, (término municipal de la Puebla de Albortón, comarca del Campo de Belchite). (Hoja 411).

Después de realizar la parada anterior, conviene efectuar un recorrido hasta el cercano pueblo de la Puebla de Albortón, a unos $6 \mathrm{Km}$, efectuaremos una nueva parada, dentro de este recorrido.

En este tramo, habremos ido encontrando afloramientos de los materiales miocénicos citados en la parada anterior. Así, en el mismo pueblo de Valmadrid habremos encontrado una pequeña explotación de materiales carbonatados, de calizas. Más adelante, casi en el lugar de la parada, habremos encontrado otra (perteneciente al municipio de Fuendetodos). 
Finalmente, al llegar al lugar de la presente parada, habremos visto importantes afloramientos de los materiales yesosos. Éstos han sido explotados en diversos lugares de esta zona.

Precisamente, en donde ahora nos encontramos, hay una pequeña explotación de yeso. Este yeso era utilizado en dos hornos de yeso, situados junto a la explotación. Los mencionados hornos, forman parte del patrimonio minero de esta comarca, a pesar de que su estado de conservación no es muy bueno.

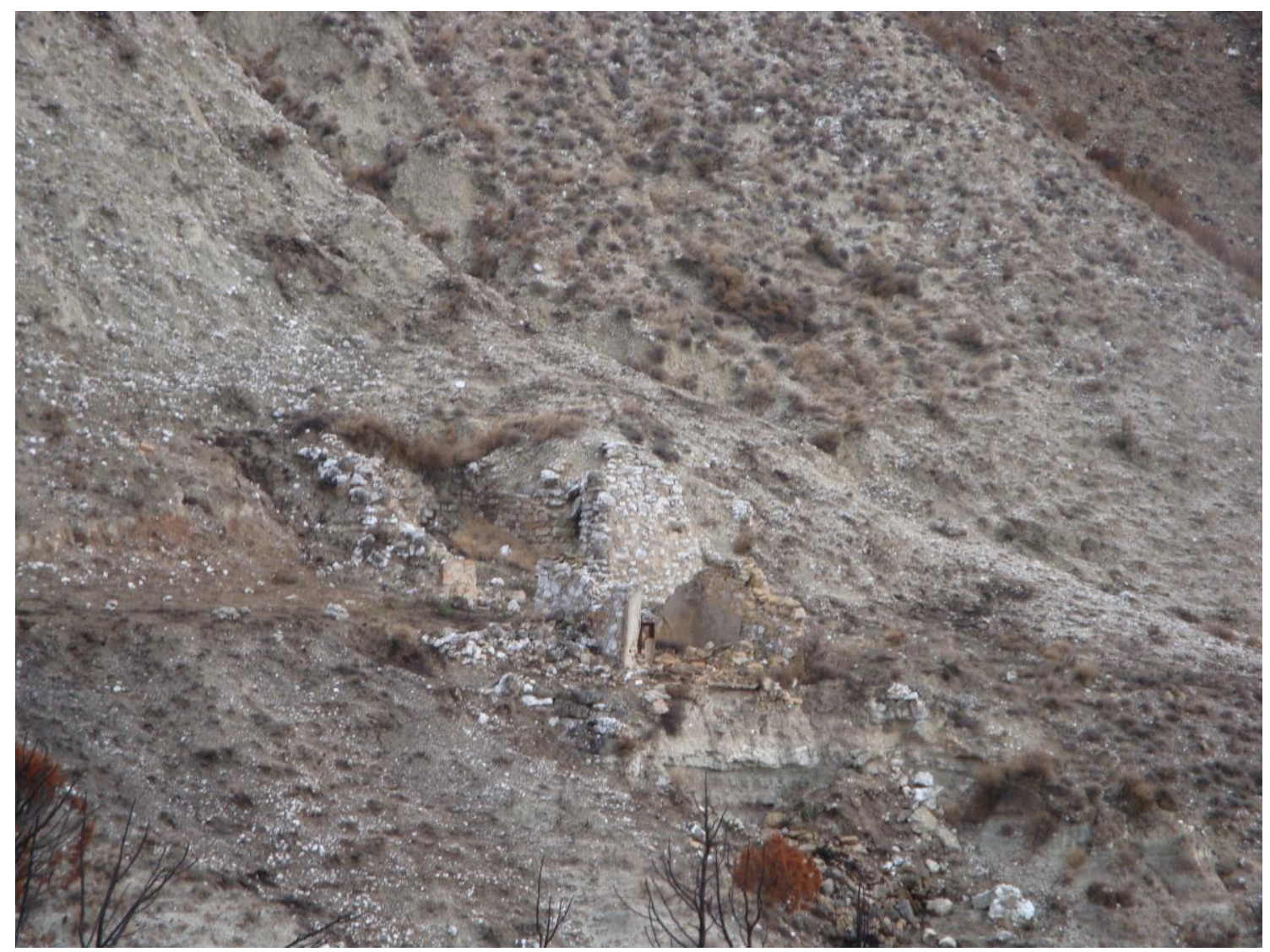

RESTOS DE UNO DE LOS HORNOS

PARADA 3. CANTERAS DE LA PUEBLA DE ALBORTÓN, (término municipal de la Puebla de Albortón, comarca del Campo de Belchite). (Hoja 412).

Después de realizar la parada anterior, conviene seguir por la carretera que conduce a la Puebla de Albortón. Luego, tras sobrepasar la población nos convendrá seguir hacia Fuendetodos. Apenas a $2 \mathrm{Km}$ de la Puebla, pasaremos muy cerca de una explotación actual de materiales carbonatados, de calizas. Ahí haremos una nueva parada, a unos $5 \mathrm{Km}$ de la anterior.

En este recorrido, efectuado íntegramente por la Depresión Geológica del Ebro, habremos encontrado afloramientos de los materiales que la rellenan, tanto del Mioceno como del cuaternario. 
En este lugar predominan los materiales carbonatados. Se trata de niveles de calizas muy blanquecinas, casi de cretas. Estas rocas están siendo explotadas en diferentes lugares de la comarca, para la obtención de cales para la construcción y para diversas industrias.

Precisamente, en el lugar de la parada hay una de estas explotaciones. Junto a ella se encuentra la planta de tratamiento. Naturalmente: para efectuar la presente parada conviene pedir autorización a la empresa explotadora.

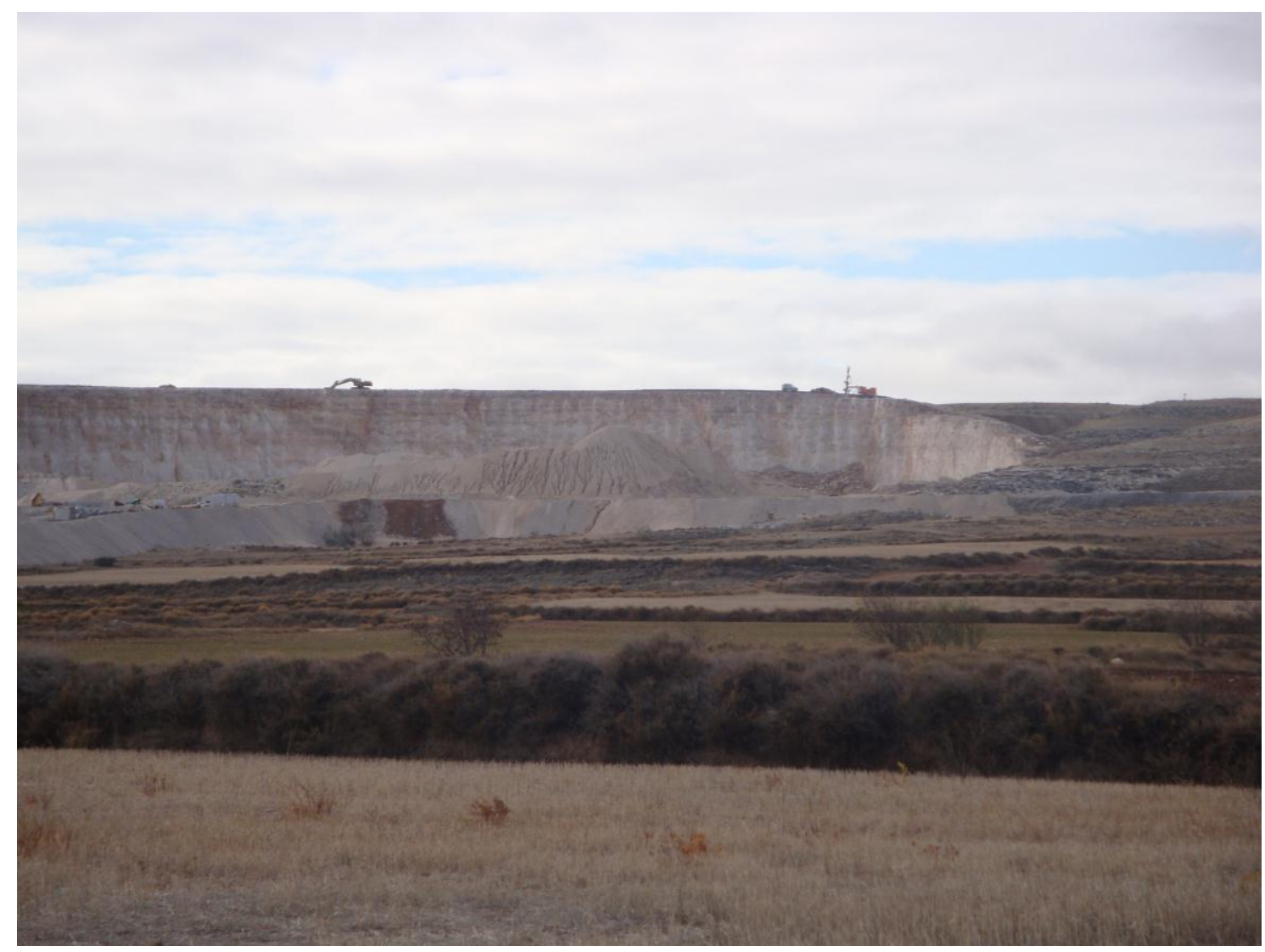

EXPLOTACIÓN DE CALIZAS DE LA PUEBLA DE ALBORTÓN

PARADA 4. ESTACIÓN DE AZUARA, (término municipal de Belchite, comarca del Campo de Belchite). (Hoja 412).

Después de realizar la parada anterior, conviene continuar por la carretera, yendo ahora siempre siempre hacía la población de Fuendetodos. Sin embargo, a unos $4 \mathrm{Km}$ de la parada anterior, encontraremos la vieja estación de Azuara del ferrocarril de Utrillas a Zaragoza (actualmente sin servicio). Ahí efectuaremos una nueva parada, dentro de este itinerario.

En este recorrido, habremos ido encontrando los materiales que hemos visto en las paradas anteriores. Estos materiales son los que rellenan por estos sectores la Depresión Geológica del Ebro, en donde seguimos estando. 
En este lugar había unas antiguas explotaciones de niveles calcolutíticos y lutíticos de tonalidades ocres. Estos materiales son también del Mioceno, como los que hemos ido encontrando a lo largo de todo el recorrido de este itinerario. Cabe decir que esta explotación está actualmente abandonada

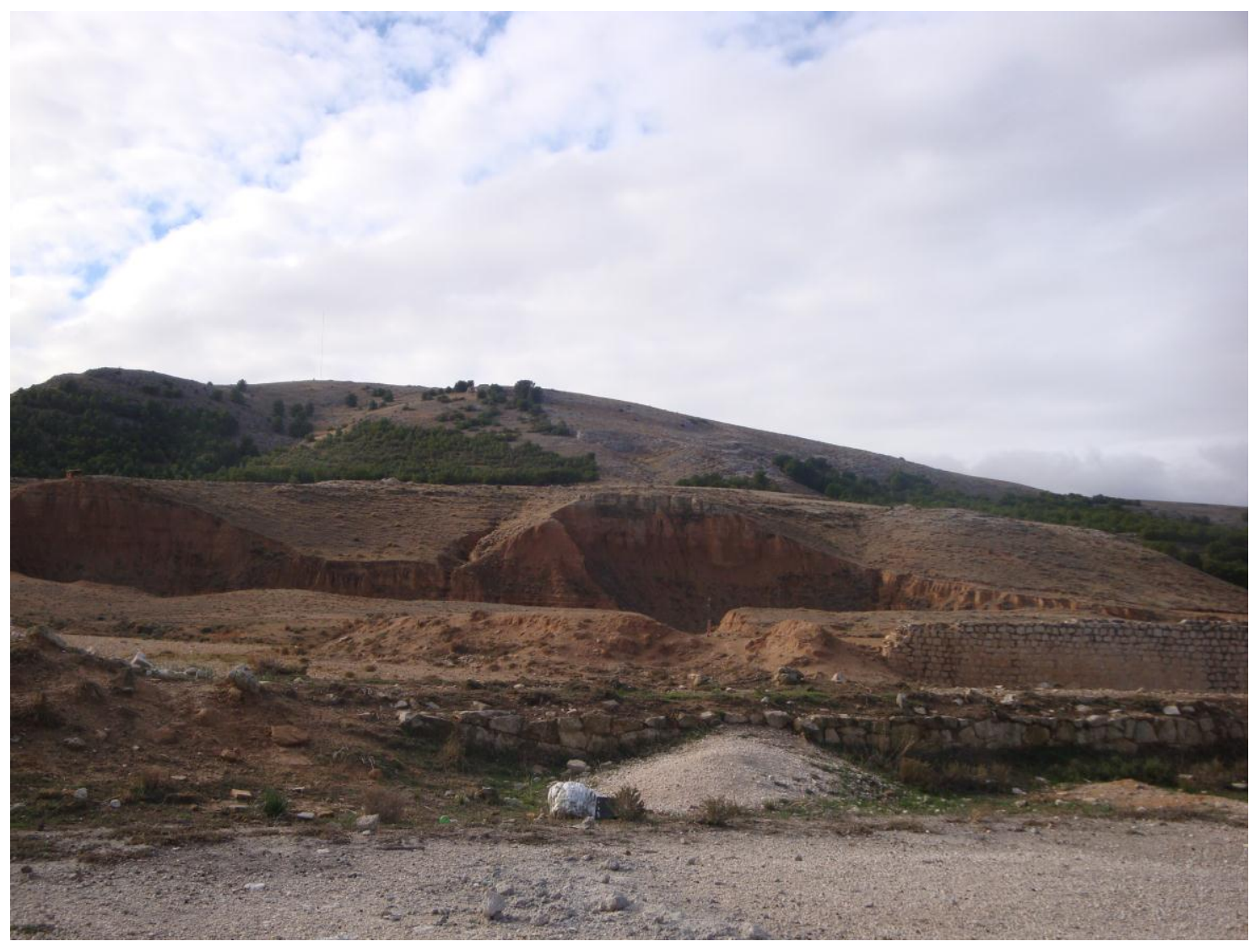

EXPLOTACIONES DE LA ESTACIÓN DE AZUARA

También cabe decir que tanto la vieja línea del ferrocarril, como la estación de Azuara, pueden considerarse como integrantes del patrimonio minero. Ambos elementos formaban parte de la línea del Ferrocarril de Utrillas a Zaragoza. Éste transportaba el carbón extraído en la cuenca de Utrillas

PARADA 5. HORNOS DE CAL DE FUENDETODOS, (término municipal de Fuendetodos, comarca del Campo de Belchite). (Hoja 412).

Tras efectuar la parada anterior, conviene dirigirnos hacía la población de Fuendetodos. Así, pronto llegaremos a la carretera que comunica Belchite con la población antes mencionada. Al encontrar-la convendrá tomarla. Al llegar, nos dirigiremos al Horno de Cal (o Calera). En este lugar realizaremos una nueva parada.

En este recorrido, como en todos los de este itinerario, habremos discurrido siempre entre afloramientos de los materiales miocénicos (y de los cuaternarios) que 
rellenan la Depresión Geológica del Ebro, en donde continuamos estando situados en este lugar.

Aquí, se halla un interesante Horno de Cal, que forma parte del patrimonio minero de esta comarca.

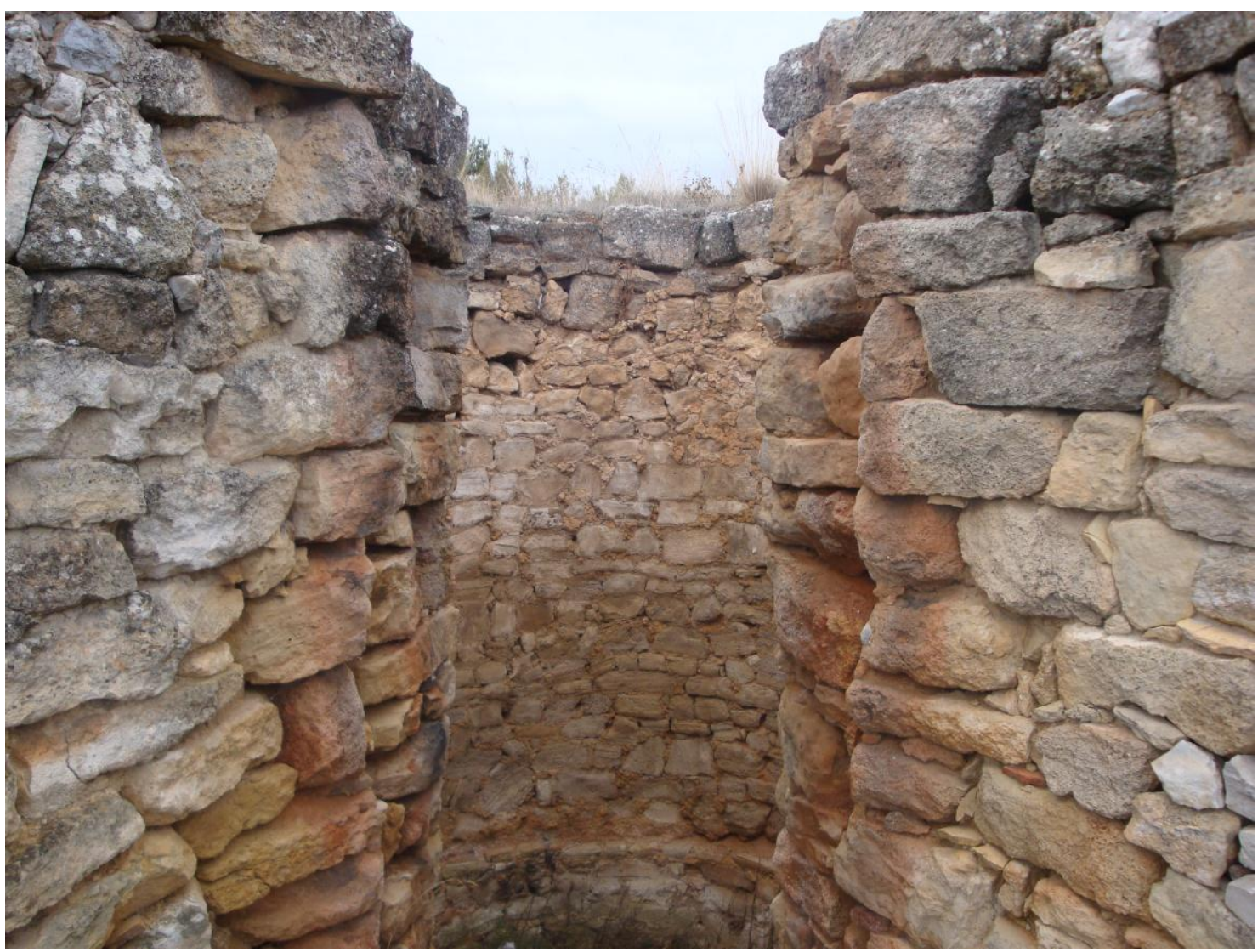

UNO DE LOS HORNOS CAL DE FUENDETODOS

Cabe decir, que en las cercanías de este Horno de Cal, similar al anterior, en el que podríamos haber realizado esta parada.

PARADA 6. CANTERAS DE CALIZA DE AZUARA, (término municipal de Azuara, comarca del Campo de Belchite). (Hoja 439).

Tras efectuar la parada anterior, conviene dirigirnos hacía la población de Azuara. Así, tras un recorrido cercano casi llegaremos a esta población. No obstante, a unos $3 \mathrm{Km}$ antes de llegar a la misma, efectuaremos una nueva parada, la última de este itinerario. Así, desde la parada anterior habremos recorrido casi $11 \mathrm{Km}$ más.

En este recorrido habremos discurrido en todo momento por la Depresión Geológica del Ebro. Así, habremos ido encontrando afloramientos de los materiales miocénicos y de los cuaternarios, como en todos los tramos anteriores del presente itinerario. 
En este lugar hay la explotación de los materiales carbonatados del Mioceno. Se trata de niveles de calizas, similares a las que hemos visto en la PARADA 3 (en la Puebla de Albortón). Como en el caso anterior, SEREQUIERE EL PERMISO DE LA EMPRESA EXPLOTADORA, PARA PODER REALIZAR LA VISITA. Como en la otra explotación, los materiales extraídos se utilizan para la fabricación de cales, tanto para la construcción como para otros usos.

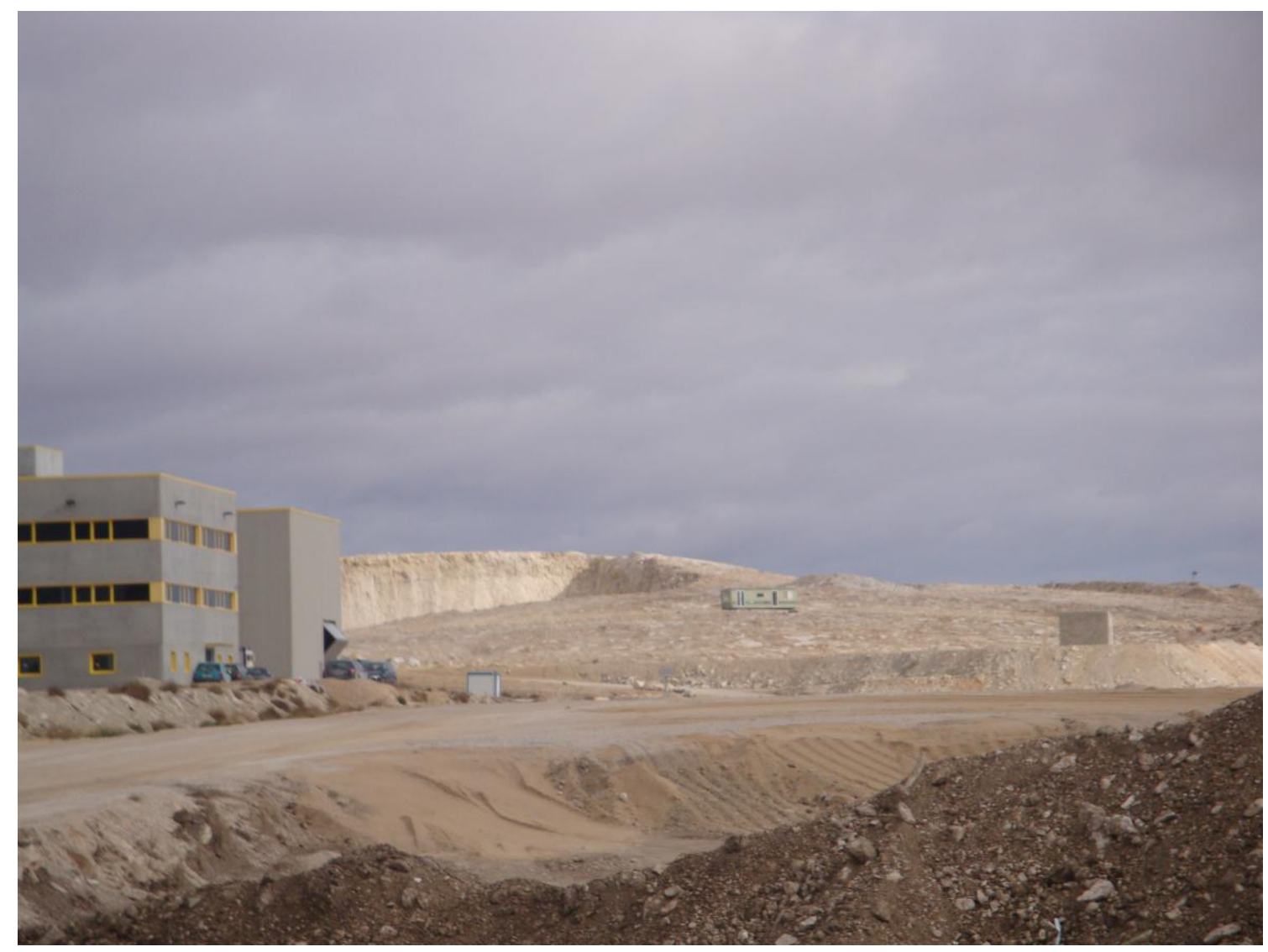

EXPLOTACIÓN DE CALIZAS EN AZUARA

\section{EN ESTE LUGAR FINALIZA EL ITINERARIO}

\section{BIBLIOGRAFÍA}

CALVO, M. (2001).- Las Minas de Sal de Remolinos, Zaragoza. Revista de Minerales, Vol. 2, no 2, pp. 3 - 22. Barcelona

CALVO, M. et altri (1988). - Minerales de Aragón, Colección Temas Geológicos, 207 Pág. Zaragoza

GOBIERNO DE ARAGÓN (2001).- Puntos de Interés Geológico de Aragón. Consejería de Medio Ambiente del Gobierno de Aragón. Zaragoza 
IGME (1972).- Mapa Geológico de España a escala 1:200.000 (Síntesis de la cartografía existente). Hoja y Memoria nº 32 (Zaragoza). Inst. Geol. Min. España

IGME (1973).- Mapa Metalogenético de España a escala 1:200.000. Hoja y Memoria $n^{\circ} .32$ (Zaragoza). Inst. Geol. Min. España

IGME (1975).- Mapa de Rocas Industriales de España a escala 1:200.000. Hoja y Memoria nº. 32 (Zaragoza). Inst. Geol. Min. España

MAESTRE, A. (1845).- Descripción geognóstica del Distrito Minero de Cataluña y Aragón. Anales de Minas, t. III. Madrid

MATA - PERELLÓ, J.; (1987).- Introducción al conocimiento de las mineralizaciones aragonesas. Mineralogistes de Catalunya, t.III, pp. 258-265. Barcelona

MATA - PERELLÓ, J.M. (1998).- Inventario Mineralógico de la comarca del Campo de Belchite, Rodeno, 25. 22 Pág. Manresa

PRAMES (2005).- Campo de Belchite. Colección RUTASCAL por Aragón. Prames, Gobierno de Aragón. 119 pag. Zaragoza. 